\title{
Energy Demand of High-Speed Connectivity Services in NG-PON Massive Deployments
}

\author{
Sofie Lambert ${ }^{(1)}$, Julio Montalvo ${ }^{(2)}$, José A. Torrijos ${ }^{(2)}$, Bart Lannoo ${ }^{(1)}$, Didier Colle ${ }^{(1)}$, Mario Pickavet ${ }^{(1)}$ \\ (1) Ghent University - iMinds, Belgium, sofie.lambert@intec.ugent.be \\ (2) Telefónica I+D Madrid, Spain
}

\begin{abstract}
Energy consumption of Next-Generation PONs is estimated in a major European city deployment scenario. For a fair comparison, Dynamic Bandwidth Allocation and Quality of Service are considered when comparing the energy demand of high speed access for the different technologies.
\end{abstract}

\section{Introduction}

Telecom market competition and an increasing demand for content-rich services such as High Definition (HD) and 3D video are driving the evolution to high speed access. At the same time, network energy consumption and its associated cost are an increasing concern for telecom operators [1].

In this paper, we evaluate the energy demand of high speed connectivity services offering up to $1 \mathrm{~Gb} / \mathrm{s}$ to each customer in Fiber To The Premises (FTTP) Next-Generation Passive Optical Networks (NG-PON). A major European city is used as deployment scenario for power consumption evaluation.

The paper is organized as follows; first, the deployment scenario, PON technologies and connectivity service model are described; next, the methodology for energy consumption analysis is reported; finally, results of energy evaluation and the main conclusions are provided.

\section{Scenario description}

City deployment scenario

An area with a radius of $70 \mathrm{~km}$ is analyzed, corresponding to a major European city surrounded by smaller towns close to the central zone. A topology with a main central area surrounded by three outer rings of less populated kernels is considered for a greenfield deployment approach, with a limited number of Central Offices (CO) per zone, see Table 1.

For each PON technology, an optimization algorithm consolidates the deployed Optical Line Terminals (OLT) as close as possible to the central zone, thus reducing the number of $\mathrm{COs}$

Tab. 1: City deployment scenario. $60 \%$ real state passed. $50 \%$ of passed homes connected.

\begin{tabular}{l|rrr} 
Zone & $\begin{array}{c}\text { Surface } \\
\left.\mathbf{( k m}^{2}\right)\end{array}$ & $\begin{array}{c}\text { \#COs } \\
\text { (max) }\end{array}$ & \#Homes \\
\hline Central & 67.27 & 1 & 564,730 \\
Ring 1 & 91.80 & 13 & 434,376 \\
Ring 2 & 113.03 & 19 & 276,217 \\
Ring 3 & 178.81 & 40 & 165,903
\end{tabular}

in the deployment. Physical reach of PONs is considered in the algorithm: the higher the reach, the lower the number of OLTs and COs in service required, thus exploiting the potential for energy efficiency of Long Reach PONs.

\section{Technologies considered}

We focus on 10G PON (E2 class as in XGPON1), XLG PON, Time-shared Wavelength Division Multiplexing (TWDM) PON, Orthogonal Frequency Division Multiplexing (OFDM) PON and Coherent Dense WDM (Co-DWDM) PON technologies, which are relevant candidates for NG-PON2 systems as recognized in the Full Service Access Network (FSAN) group. In order to evaluate the energy efficiency of long reach in PONs, we have selected the maximum budget classes for each technology, listed in Table 2. We consider Gigabit/Ethernet PON (GPON/EPON) with B+ optics as legacy technology for reference. Only passive longreach approaches are considered in order to avoid active elements in the Optical Distribution Network (ODN). We only envisage power splitter-based architectures, since these are compatible with legacy ODNs.

\section{Dynamic Bandwidth Allocation and User behavior models}

The statistical gain in Time Division Multiple Access (TDMA) technologies is considered, assuming mechanisms are available both in downstream and upstream direction for offering each active user a variable bandwidth in the PON interface, with dynamic adaptation to the total user demand versus time (Dynamic Bandwidth Allocation).

If $N$ users are connected to an OLT PON interface, the minimum bandwidth that can be offered to each user is

$$
B_{\min }=\frac{\text { PON bandwidth }}{N}
$$

This can be guaranteed $100 \%$ of the time. However, users are not active all the time. The probability that each user demands bandwidth from the PON is quantified by the parameter $p_{\text {act }}$, the active user probability. 
Tab. 2: PON technologies: performance and power consumption parameters [2]. Co-DWDM PON estimations scaling power processing for OLT + amplifier and assuming same ONU power consumption as XLG PON.

\begin{tabular}{l|rrrrr} 
Technology & $\begin{array}{c}\text { Bandwidth } \\
\text { DS/US (Gb/s) }\end{array}$ & Max. budget & PONs/ chassis & $\begin{array}{c}\text { power / OLT } \\
\text { port (W) }\end{array}$ & $\begin{array}{c}\text { power / ONU } \\
\text { (W) }\end{array}$ \\
\hline G/E PON (B+) & $2.5 / 1.25$ & $28 \mathrm{~dB}$ & 128 & 2 & 5.1 \\
10G PON (E2) & $10 / 2.5$ & $35 \mathrm{~dB}$ & 64 & 5 & 7.1 \\
XLG PON & $40 / 10$ & $31 \mathrm{~dB}$ & 32 & 17 & 14.6 \\
TWDM PON & $4 \times 10 / 4 \times 2.5$ & $38.5 \mathrm{~dB}$ & 32 & 20 & 7.1 \\
OFDM PON & $40 / 10$ & $34.5 \mathrm{~dB}$ & 32 & 67.5 & 11.1 \\
Co-DWDM PON & $160 / 160$ & $43 \mathrm{~dB}$ & 32 & 89.2 & 14.6
\end{tabular}

Considering the statistical user demand, the service provider can offer much higher bandwidths up to:

$$
B_{\text {max }}=\frac{\text { PON bandwidth }}{k} \geq B_{\text {min }}, \quad 1 \leq k \leq N
$$

These values are not guaranteed $100 \%$ of the time, but a lower percentage $p_{\text {avail }}$ (availability, $\%$ ), equal to the probability that the number of active users on a PON interface is $k$ or less [3].

Connectivity service model and Quality of Service (QoS) quantification

We consider a connectivity service with a certain target bandwidth, $B_{\text {target }}$, which is requested to the PON when a user is active. Each user is active $p_{\text {act }} \%$ of time. Depending on the number of active users $k$ at a given point in time, $B_{\max }$ can be lower than $B_{\text {target, }}$ in which case the target bandwidth can't be offered ( $p_{\text {avail }}<100 \%$ ). We assume a single service priority for all users. As NG-PON2 protocols are still not closed, only raw bit capacity has been quantified without considering protocol efficiency.

QoS requirements are quantified using two parameters: $p_{\text {avail }}(\%)$, minimum percentage of time that the target bandwidth is available for each connected user; and MPL (Maximum Packet Loss), the maximum ratio of packets discarded over packets offered in the uplink interface of a PON chassis (from the OLT to the aggregation network).

This service model and the QoS quantification provide a fair analytical framework in order to compare each of the PON technologies.

\section{Energy efficiency analysis: methodology}

We consider the power consumption at the customer side (ONU, values in Tab. 2) and at the operator side (CO). The latter is the sum of the following power consumption contributions:

- OLT PON interfaces

- Layer 2 switching (chassis back-panel), considering $1 \mathrm{~W} / \mathrm{Gb} / \mathrm{s}$

- Uplink ports, combining ports with capacities 1, 10, 40, 100, 400 and $1000 \mathrm{~Gb} / \mathrm{s}$, which consume 7, 30, 70, 130, 300 and $550 \mathrm{~W}$.

Note that energy saving mechanisms like sleep modes are not considered in this work. Further, power consumption values do not include AC/DC conversion and cooling overhead.

We determine the equipment inventory, which is needed to calculate the power consumption of the massive deployment, as follows. First, for each connectivity service with a fixed user demand profile ( $p_{\text {act }}$ and $B_{\text {target }}$ ) and fixed QoS parameters ( $p_{\text {avail }}$ and $\left.M P L\right)$, the optimal split ratio and uplink port capacity are calculated for each PON technology. We assume a maximum split ratio of $1: 256$, with additional limitations posed by the max. budget. Next, the split ratio is used as input for the deployment algorithm that calculates the equipment inventory.

\section{Results and discussion}

The bars in figs. 1-3 show the power consumption at the $\mathrm{CO}$. Although the discussion focuses on the $\mathrm{CO}$, we include the values of $\mathrm{CO}+\mathrm{ONU}$ (markers, note the different scale) for completeness. The split ratio for each technology is shown above the bars in all the figures.

Fig. 1 summarizes the results for low user activity $\left(p_{a c t}=10 \%\right)$. L2 switching is the main contributor to the power consumption at the $\mathrm{CO}$, since it scales with capacity (independent of the actual demand) and the networks are overdimensioned for low user activity. In fig. 1, each technology can be used at its highest possible split ratio (limited by max. budget), even for high values of $B_{\text {target }}$ As the split ratios remain constant, so do the number of PON ports and switching capacity and their power consumption. For increasing $B_{\text {target }}$ and decreasing MPL, higher uplink capacities are required, resulting in higher power consumption in the uplink, but the overall impact on power consumption is relatively small.

The results for medium user activity $\left(p_{\text {act }}=20 \%\right)$ are given in Fig. 2. In this case, lower-capacity PONs require lower split ratios in order to support high speed connectivity with high QoS requirements (increasing $p_{\text {avail }}$ ). The power per user at the $\mathrm{CO}$ for these technologies increases significantly since the equipment is shared by 


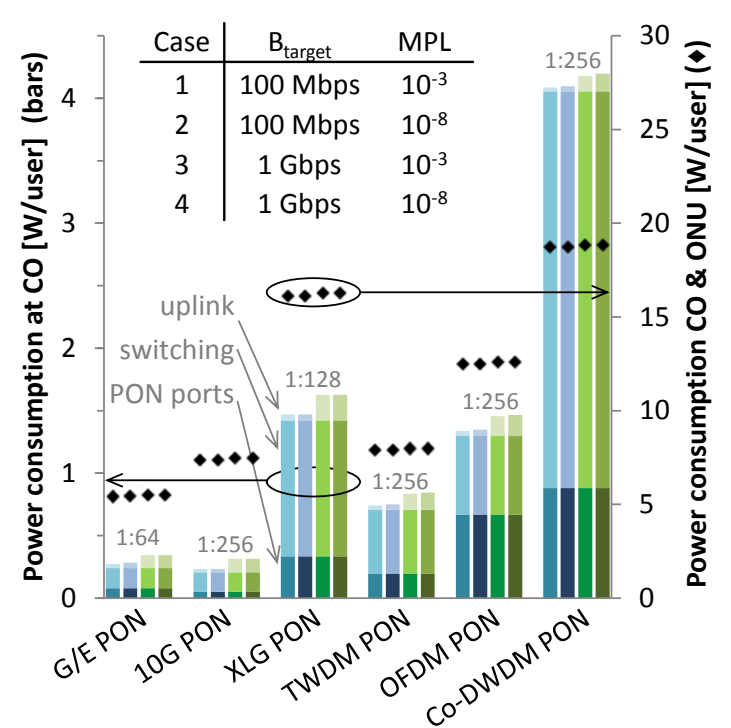

Fig. 1: Power consumption for low user activity: $p_{\text {act }}=10 \%, p_{\text {avail }} \geq 20 \%$. Cases $1-4$ are shown from left to right for each technology.

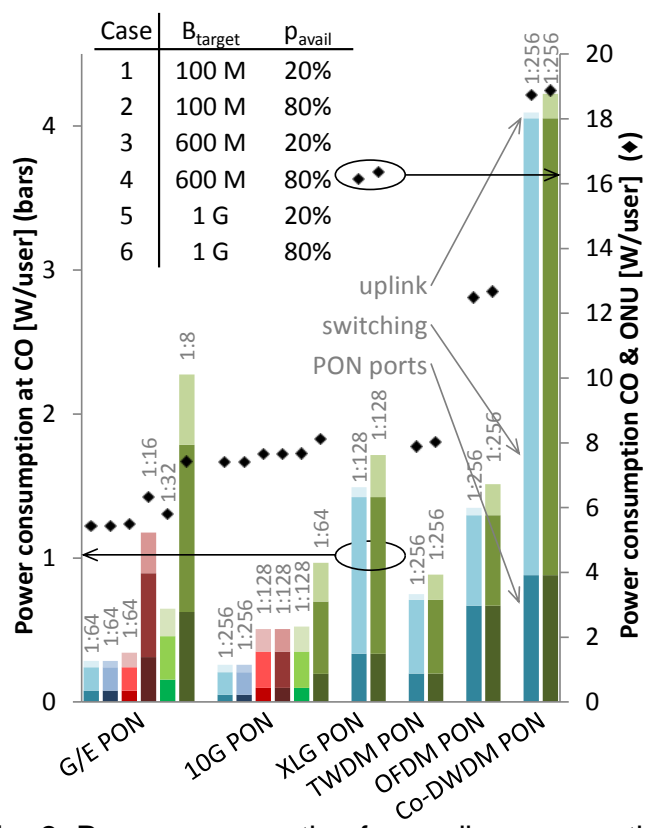

Fig. 2: Power consumption for medium user activity: $p_{\text {act }}=20 \%, M P L=10^{-5}$. Cases 1-6 are shown from left to right for G/E PON and 10G PON; only cases $1 \& 6$ are shown for NG-PON2 technologies since intermediate cases are similar.

fewer users. In contrast, the increase in power consumption for higher-capacity NG-PON2 technologies is small, since they can still be used at their highest split ratio in all cases.

Fig. 3 shows the results for high user activity $\left(p_{\text {act }}=50 \%\right)$. For high speed connectivity (high $B_{\text {target }}$, the PON ports and uplink start to take up a bigger share of the power consumption since lower split ratios are required. Split ratios for the lower capacity technologies as low as 1:8 are required in some cases, making them unsuitable for practical deployments. For the highest connectivity speed $\left(B_{\text {target }}=1 \mathrm{~Gb} / \mathrm{s}\right)$, TWDM and XLG PON become the more energy efficient

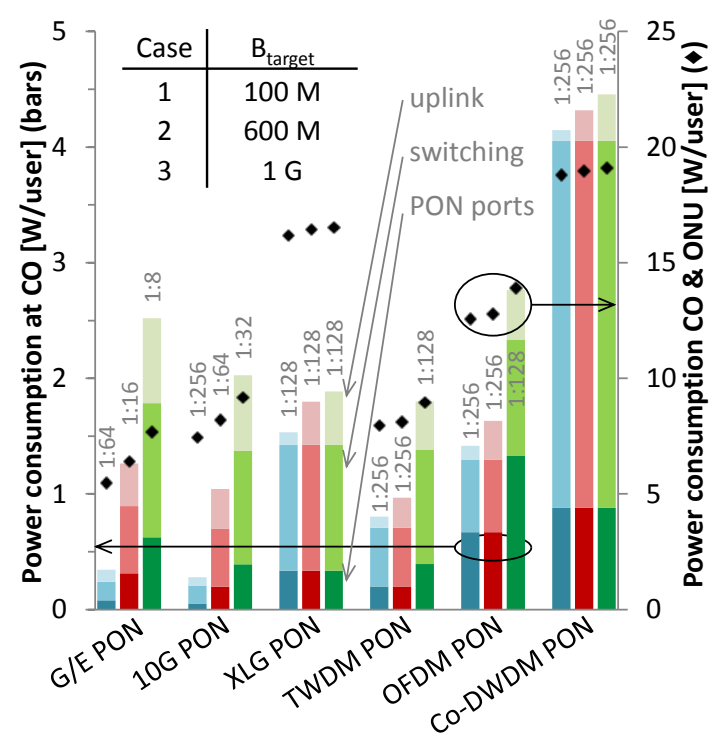

Fig. 3: Power consumption for high user activity: $p_{\text {act }}=50 \%, \mathrm{MPL}=10^{-5}, p_{\text {avail }} \geq 20 \%$. Cases $1-3$ are shown from left to right for each technology.

technologies at the CO. Due to their high capacity, L2 switching power consumption is still the main contributor for these technologies.

Note that, for all cases, our estimations show that Co-DWDM PON demands more energy consumption; this is because bandwidth is not shared among ONUs, leading to a 100\% guarantee of the target bandwidth (or even higher) with the lowest latency. This can be a requirement for certain applications such as mobile backhauling or business services. When targeting residential customers, a form of TDM (sharing a wavelength and processing resources with $>1$ customers) could be very beneficial for enhancing energy efficiency in Co-DWDM PON.

\section{Conclusion}

We have demonstrated that standardized technologies G/E PON cannot offer high speed services up to $1 \mathrm{~Gb} / \mathrm{s}$ per customer with legacy ODN (1:32-64 split ratio) satisfying the same QoS requirements and achieving the same energy efficiency as NG-PON2 technologies. With the available power estimations, NG-PON2 technologies such as TWDM PON become the most energy efficient options for high speed connectivity services.

\section{Acknowledgements}

This research has received funding from the EU FP7 projects TREND (ICT-257740) and DISCUS (ICT-318137). The first author is funded by an IWT-Vlaanderen grant.

\section{References}

[1] S. Lambert et al., Opt. Express, 20, 26 (2012).

[2] B. Skubic et al., IEEE Comm. Mag., 50, 1 (2012).

[3] J. Segarra et al., ICTON '11, Tu.A6.4 (2011). 